\title{
Progress in Pulmonary Rehabilitation of Patients Undergoing Thoracoscopic Radical Resection of Lung Cancer
}

\author{
Fu-Rong $\mathrm{Li}^{1}$, Lin $\mathrm{Han}^{2, *}$, $\mathrm{Li} \mathrm{Li}^{2}$ \\ ${ }^{1}$ Shaanxi University of Chinese Medicine, Xianyang, Shaanxi, China. \\ ${ }^{2}$ Affiliated Hospital of Shaanxi University of Traditional Chinese Medicine, Xianyang, Shaanxi, China.
}

How to cite this paper: Fu-Rong Li, Lin Han, Li Li. (2021) Progress in Pulmonary Rehabilitation of Patients Undergoing Thoracoscopic Radical Resection of Lung Cancer. International Journal of Clinical and Experimental Medicine Research, 5(1), 33-40.

DOI: 10.26855/ijcemr.2021.01.007

Received: December 12, 2020

Accepted: January 8, 2021

Published: January 18, 2021

*Corresponding author: Lin Han, Affiliated Hospital of Shaanxi University of Traditional Chinese Medicine, Xianyang, Shaanxi, China.

Email: 786894316@qq.com

\begin{abstract}
With the aggravation of population aging, the incidence of lung cancer is increasing year by year. As the preferred treatment for lung cancer, radical resection of lung cancer is also developed from traditional thoracotomy to thoracoscopic surgery relying on the development of minimally invasive surgery. Pulmonary rehabilitation is a comprehensive rehabilitation treatment program that can promote pulmonary function rehabilitation of patients after radical resection of lung cancer. It mainly adopts measures such as sports training, drug rehabilitation, nutritional rehabilitation, pulmonary rehabilitation education and psychosocial support to improve patients' exercise tolerance, pulmonary function and quality of life. In this paper, the recent clinical studies on pulmonary rehabilitation after radical resection of lung cancer by thoracoscopic surgery were reviewed to explore the future development direction of pulmonary rehabilitation after radical resection of lung cancer.
\end{abstract}

\section{Keywords}

Thoracoscopic Surgery, Radical Resection of Lung Cancer, Postoperative Pulmonary Rehabilitation

The 2018 Global Cancer Annual Report shows that lung cancer is the cancer with the highest incidence and mortality in the world, accounting for $11.6 \%$ of new cancer cases and $18.4 \%$ of total cancer deaths, respectively [1]. More than $80 \%$ of the patients with lung cancer are aged 60 years or above, and the majority are males [2]. Lung cancer is the most common malignant tumor in China. The incidence of lung cancer is increasing year by year with the aggravation of population aging, which poses a serious threat to the physical and mental health of residents. In recent years, thoracoscopic minimally invasive surgery has been gradually accepted by the public for its advantages of less trauma, faster recovery, and better tolerance after chemotherapy. Both domestic and foreign guidelines recommend thoracoscopic radical resection of lung cancer as a treatment for early lung cancer [3-4]. Lung cancer radical prostatectomy is the best way to treatment of early lung cancer treatment, mainly including the lymph node lobectomy and, although surgery can prolong survival in patients with lung cancer, but in single ventilation and tracheal catheter anesthesia and postoperative lung volume reduction, postoperative wound pain, the influence of both the respiratory function in patients with impaired, sports endurance is low and the quality of life [5-6]. Lung cancer rehabilitation, as a new field, has been widely concerned by scholars at home and abroad. A number of studies have shown that early postoperative pulmonary rehabilitation can help patients rebuild lung function by improving patients' cardiopulmonary and peripheral muscle endurance [7-9]. At present, pulmonary rehabilitation 
measures for lung cancer mainly refer to mature treatment programs of COPD pulmonary rehabilitation, and the application of pulmonary rehabilitation after lung cancer surgery is at the preliminary exploration stage. Therefore, this paper summarizes the research progress of pulmonary rehabilitation after thoracoscopic radical resection of lung cancer, in order to provide scientific basis for clinical nursing practice.

\section{Concept of pulmonary rehabilitation}

The term "pulmonary rehabilitation", as early as 1974 by ATS (ATS) is proposed, and its concept after several modifications, founded in 2013 by ATS and European respiratory society (ERS) to establish a consensus, points out that pulmonary rehabilitation is based on the comprehensive evaluation of patients, for patients with tailor-made a set of comprehensive intervention treatment, including but not limited to sports training, education and behavior change, aimed at improving mental and physical health and patients with chronic respiratory system disease and insist for a long time to promote health behavior [10]. At present, pulmonary rehabilitation after radical resection of lung cancer mainly refers to COPD, including exercise training, drug rehabilitation, nutritional rehabilitation, pulmonary rehabilitation education and psychosocial support.

\section{Significance of pulmonary rehabilitation after thoracoscopic radical resection of lung can- cer}

Although thoracoscope surgery with small incision, less intraoperative visibility, ribs, pull, muscle damage and intercostals nerve damage small, short postoperative chest drainage time and less traffic and shorter postoperative hospital stay, and many other advantages [11], but art is still hard to avoid in the chest wall muscle damage, and lobectomy patients after lung volume is reduced, increased airway secretions, and obvious chest pain, lungs breathing exercise capacity is low, easily happened lung infection, atelectasis, ARDS, respiratory failure and other complications [12]. In addition, unilateral lung ventilation is often performed through bronchial tubes during radical lung cancer surgery. During the process of unilateral lung ventilation, the patients' thoracotomy side lung completely shrinks, resulting in decreased blood shunt and ventilation blood flow ratio within the lung, and relative hyperventilation of the contralateral lung, which increases the risk of postoperative lung injury. Under hypoventilation, cell ischemia and hypoxic destruction and injury may occur, leading to various pulmonary complications in patients [13-14]. Therefore, postoperative pulmonary rehabilitation is particularly important, the purpose of which is to enable patients to recover lung function and physical and mental state as soon as possible, maximize the improvement of patients' activity ability, and improve the quality of life.

\section{Measures and timing of pulmonary rehabilitation after thoracoscopic radical resection of lung cancer}

\subsection{Pulmonary rehabilitation measures for patients after thoracoscopic radical resection of lung can- cer}

\subsubsection{Sports training}

\subsubsection{Upper limb exercise training}

Upper limb exercise training mainly improves the patient's forearm movement ability, improves activity endurance and reduces ventilation demand by enhancing the body's adaptability to upper limb movement. Common training methods include arm movement, fist movement, two upper limbs circling, upper limb weight lifting such as dumbbell lifting, arm power bicycle. The common methods of grip strength training include towel twisting training and grabbing objects as hard as possible, which can increase the strength of patients' forearm extension and flexor muscles [15]. Kong et al. [16] asked the patients after lung cancer surgery to use a gym bar to do exercises in all directions that were above the shoulder height, and held dumbbells $(0.5-3 \mathrm{~kg})$ to do exercises that were higher than the shoulder height. After 12 weeks of exercise, the patients' exercise ability was improved. Fan Zerong et al. required that the affected arm should carry a weight of $0.25 \mathrm{~kg}$ (half bottle of mineral water) and the healthy arm should carry a weight of $0.5 \mathrm{~kg}$ ( 1 bottle of mineral water) when the patient was lifting the arm, and the patient should coordinate breathing with arm lifting, lasting about 15min each time [17].

\subsubsection{Lower limb exercise training}

Lower limb movement is a routine item of postoperative pulmonary rehabilitation. Patients with lung cancer radical surgery should be encouraged to bed early, by increasing the large muscle groups of activities to improve physical muscle function, improve the ability to walk, promote sports endurance, also can increase the alveolar 
ventilation, tidal volume to improve cardiopulmonary function [18], common training methods are walking walking, jogging, climbing stairs, tablet and power cycling, etc. Chen Xiang et al. [19] suggested that on the first day after surgery, the rehabilitator and the nurse jointly guide the patient to ride a bicycle on the back with a foot pedal trainer, 3 times a day, 10 to 20 minutes each time, to strengthen the exercise of lower limbs, and the patient should stop immediately if he feels chest tightness and shortness of breath. Vagvolgyi et al. [20] advocate that patients with lung cancer participate in individualized, continuous or intermittent cycle and/or treadmill training, 2 to 3 times a day, each time lasting 10 to 30 minutes, with a maximum intensity of $60 \%$ to $80 \%$.

\subsubsection{Respiratory training}

Respiratory muscle weakness is the main factor for the difficulty in coughing up sputum and dyspnea in patients after radical resection of lung cancer. Respiratory training can improve patients' respiratory function and relieve dyspnea. Common training methods include lip contraction breathing, abdominal breathing, balloon blowing, breathing exercises, breathing apparatus training, chest expansion exercise training and diaphragm electrical stimulation. Studies have shown that guiding patients to do abdominal breathing and labial contraction breathing after surgery is beneficial to increase the volume of air and alveolar ventilation, and can promote the recovery of lung function [21-22]. In Huang Yuxian's study [23], patients exercised their respiratory function with the help of a breathing trainer, which significantly reduced the incidence of postoperative respiratory complications and improved postoperative lung function. Chest dilation exercise training can prevent pulmonary dilatation insufficiency caused by defensive muscle contraction induced by postoperative pain [24]. Wang Hao [25] applied diaphragmatic electrical stimulation training, a new respiratory training method, to patients after radical resection of lung cancer, which can control the patient's respiratory frequency and depth, and has achieved good benefits in improving the patient's lung function. In addition, Traditional Chinese medicine also plays an important role in lung rehabilitation. Respiration exercises with Traditional Chinese medicine characteristics mainly include meridian-Collaterals exercise and health-preserving respiration exercises. The main points of the breath exercise of meridian and collaterals are pointing, rubbing the chest, beating the chest and beating the back, and expanding the chest. The common exercises of the breath exercise of traditional Chinese medicine for health preservation include six-character formula, Guiyuangong, Loosing and nourishing the body in quietness, Baduan Jin, and the breath exercise of standing piles [26-27].

\subsubsection{Drug rehabilitation}

After radical resection of lung cancer, patients often have pulmonary manifestations such as sputum retention, respiratory membrane edema, airway spasm and pneumonia, etc., which need to be assisted by the administration of expectorant, glucocorticoid, bronchus dilator and antibiotics, etc. (1) Expectorant: ambroxol hydrochloride is a commonly used drug, which can reduce the viscosity of sputum and help expectorant. (2) Glucocorticoid: Glucocorticoid is a widely used non-specific anti-inflammatory drug, which can effectively inhibit inflammatory response, reduce respiratory membrane edema, and improve respiratory symptoms [28]. (3) Bronchodilator: The commonly used drug is tiotropium bromide, which can relieve airway spasm in patients with lung cancer, reduce airway resistance, improve the over-inflated lung state, reduce sputum production and lung retention, and thus reduce the incidence of postoperative pulmonary complications [29]. (4) Anti-infection: pneumonia is the main cause of death of lung cancer patients after surgery, so should be prophylactic use of antibiotics in order to avoid the occurrence of postoperative pneumonia, control postoperative pulmonary infection, eliminate inflammation.

\subsubsection{Nutritional rehabilitation}

Nutritional rehabilitation is a part of pulmonary rehabilitation. Nutritional support can effectively ensure the metabolic needs of cells in the body and improve immunity. After radical cure of lung cancer, patients can be given intravenous high nutrition, amino acids, fat milk and albumin are injected intravenously for nutritional support, and patients can be instructed to eat a light, digestible, high-calorie, high-protein diet after anesthesia. Studies have shown that [30] early postoperative enteral nutrition can effectively improve postoperative nutrition level and reduce the risk of pulmonary infection in patients with lung cancer.

\subsubsection{Pulmonary rehabilitation education}

Pulmonary rehabilitation education includes instruction on airway clearance techniques, splint cough, abdominal breathing, lip reduction breathing, upper and lower limb exercise, walking exercise, and stair exercise. In a randomized controlled experiment conducted by Jeong et al. [31], pulmonary rehabilitation education was provided for patients in the experimental group and comprehensive management advice was provided for patients in the control group. After 4 weeks, respiratory muscle strength and dyspnoia of patients in the experimental group were signifi- 
cantly improved compared with those in the control group. Selzler et al. [32] proposed remote pulmonary rehabilitation education, which provides patients with information services related to disease management based on the network platform, reducing the cost of medical care and thereby reducing the economic burden of patients.

\subsubsection{Psychosocial support}

Patients after lung cancer surgery mostly have psychological disorders, such as fear of pain caused by surgical trauma and other adverse stimuli, anxiety and worry about prognosis, and there is a wrong understanding of the disease. Medical staff and family members should encourage patients to fight against the disease and provide comprehensive psychosocial support. Studies have shown that personalized and multidisciplinary rehabilitation plan formulated by medical staff, patients and family members in cooperation with each other can stabilize the mood of patients after lung cancer surgery and improve their compliance with rehabilitation exercise [33-34].

\subsection{Pulmonary rehabilitation opportunity of patients after thoracoscopic radical resection of lung cancer}

At present, there are no uniform standards and norms for the time, frequency and intensity of pulmonary rehabilitation training for patients after radical resection of lung cancer. Relevant studies have shown that the duration of pulmonary rehabilitation training after lung cancer surgery is 1 week after surgery [35]. Other studies have pointed out that the duration of pulmonary rehabilitation training after surgery is 12-24 weeks after surgery [36-37]. Systematic analysis shows that the duration of pulmonary rehabilitation training after lung cancer surgery is mostly 4-8 weeks after surgery, but further study and confirmation are still needed [38]. Based on a comprehensive analysis of a number of interventional studies, it was found that the earlier pulmonary rehabilitation training started after lung cancer surgery, the more beneficial it was to enhance athletic ability, improve quality of life and reduce postoperative pulmonary complications. Therefore, patients with lung cancer can be encouraged to do respiratory function training and guided to take slow deep breaths (once in the morning and once in the evening, 20-30 times each time) after anesthesia and wake up after surgery. 2-3d after surgery, patients were instructed to do abdominal breathing, effective cough and sputum, horizontal or comfortable semi-supine breathing exercises (once a day, 10-15min each time), and upper and lower limb exercises (three times a day, 10-15min each time) [22]. 4-7d after the removal of thoracic drainage tube, the intensity of breathing exercise can be gradually increased, and patients are encouraged to sit up and hang their lower limbs from the bedside $\rightarrow$ sit quietly in a chair $\rightarrow$ get out of bed and stand $\rightarrow$ walk around the bed $\rightarrow$ walk in the ward and perform standing breathing exercise [39]. 8-10d after the operation, the daily walking distance of the patient could be gradually extended, and the daily standing breathing exercise could be carried out continuously (10-15min each time) to increase the number and time of exercise, and stair climbing training could be carried out according to the patient's recovery. Four weeks after the operation, with the patient's condition improving day by day, high-intensity endurance and strength training can be gradually increased.

\section{Effect evaluation of pulmonary rehabilitation after thoracoscopic radical resection of lung cancer}

\subsection{Objective effect evaluation}

\subsection{1 restingpulmonary functiontest, $P F T$}

The commonly used indicators are: forced expiratory volume in one second (FEV1), forced vital capacity (FVC), and diffusion capacity for carbon monoxide of the lung (DLCO). Among them, FEV1 and DLCO are not only objective indicators for preoperative evaluation of patients' respiratory tolerance to lung cancer surgery, but also independent indicators for prediction of postoperative complications [40].

\subsubsection{Exercise test}

1) The cardiopulmonary exercise experiment (CPET)

Commonly used indicators include VO2max, CO2 emissions, VE per minute, MVV, electrocardiography, blood pressure, peak Oxygen consumption (VO2peak). CPET can increase the load of the cardiopulmonary system and oxygen delivery system through a certain amount of exercise, which can not only help to guide the evaluation of postoperative cardiopulmonary rehabilitation training, but also simulate the load exerted on patients by surgery to a certain extent, and contribute to the preoperative evaluation of patients' surgical tolerance [41].

2) Six-minute walk Distance (6MWD)

6MWD determines the cardiopulmonary function of a patient according to the walking distance within a limited time, and the lower the level is, the worse the cardiopulmonary function is [15]. 
At the end of $6 \mathrm{~min}$, the walking distance is recorded, $<300 \mathrm{~m}$ is level $1,300-374.9 \mathrm{~m}$ is level $2,375-450 \mathrm{~m}$ is level 3 , and $>450 \mathrm{~m}$ is level 4 .

3) Stair Climb test (SCT)

SCT has been used in clinical practice for a long time as a cardiopulation exercise test. The number of stairs can reflect the patient's maximum oxygen consumption (VO2max) to a certain extent, but there is no unified standard on the height of floors and steps. Kubori et al. [42-43] used the stair climbing test to evaluate the improvement of exercise ability of lung cancer patients after lobectomy. The staircase is composed of 36 stairs, with each stair consisting of 20-31 steps, and the height of each stair is $0.18-0.19 \mathrm{~m}$.

4) Shuttle walk test (SWT)

Refers to the patient walking back and forth within a distance of $10 \mathrm{~m}$, with the pace following the established fixed rhythm and gradually increasing speed until the patient has difficulty in breathing or cannot continue walking [44]. During the SWT test, the patient's walking distance, dyspnea index score, recovery time and reason for cessation of exercise were recorded, and blood oxygen saturation was measured every 30s.

\subsubsection{Evaluation of pulmonary secretions}

The common clinical evaluation methods include pulmonary auscultation, fiber electron microscopy and ultrasound examination. Lung auscultation can determine the concentration of secretions; the quality and quantity of the patient's pulmonary secretions can be directly observed by fiberoptic microscopy, and alveolar lavage can also be performed by fiberoptic microscopy to remove long-term residual secretions in the lungs ${ }^{[45]}$.The bedside evaluation of the patient may be performed by ultrasound examination.

\subsubsection{Incidence of postoperative pulmonary complications}

Pulmonary complications such as respiratory dysfunction, pulmonary infection, atelectasis and pulmonary embolism are also indicators to evaluate the effectiveness of pulmonary rehabilitation after radical resection of lung cancer.

\subsection{Subjective effect evaluation}

\subsubsection{Quality of life evaluation}

Quality of life is an important indicator to evaluate the effectiveness of postoperative pulmonary rehabilitation, and is also one of the ultimate indicators of clinical rehabilitation treatment, covering 5 aspects including physical health, mental health, social function, daily life activities and good sense of self. The quality of life scale (EORTC-QLQ-C30) and the Lung Cancer Specific Scale (EORTC-QLQ-LC13) of the European Organization for Research and Treatment of Cancer (EORTC) can be used to evaluate the quality of life [46].

\subsubsection{Evaluation of dyspnea index}

The main factor affecting the postoperative life quality of patients with lung cancer is dyspnea, and postoperative pulmonary rehabilitation training can reduce the degree of dyspnea of patients. Evaluation tools include the most commonly used Brog score and the most sensitive BODE index score.

(1) Brog score (10-point scale): The Brog score is often used to evaluate the degree of dyspnea. The score range is $0-10,0$ means no dyspnea, 10 means extreme dyspnea, the higher the score is, the more severe the dyspnea is; (2) the BODE index score (the body mass, airflow obstruction, dyspnea, and exercise capacity index): BODE index including lung function, dyspnea, athletic ability and body mass index (bmi) four aspects. Candemir et al. showed that BODE index could determine the curative effect of pulmonary rehabilitation [47].

\section{Summary}

Lung cancer treated with thoracoscope technology is the primary method for early lung cancer patients the best operation, the postoperative pulmonary rehabilitation exercise training, medicine, nutrition, rehabilitation, pulmonary rehabilitation education and social psychological support, etc., with lifting movement endurance, improve patients' respiratory function, reduce pulmonary complications, relieve anxiety, depression and other negative emotions, shorten the length of hospital stay and reduce expenses related to health and other advantages. But lung pulmonary rehabilitation as an emerging field, domestic clinical literature reported less, has the following problems need to be further discussed: (1) The lung cancer effect a radical cure postoperative pulmonary rehabilitation mainly refer to the COPD pulmonary rehabilitation training method, will be the same without pulmonary rehabilitation measures and effect evaluation screening and identification of patients with lung cancer radical surgery applications, lack of specificity, and its effectiveness still needs more clinical research to explore. (2) Most of the domestic and 
foreign studies on lung cancer rehabilitation are single-center small sample trials. There are no unified standards on when to start lung rehabilitation, evacuation time, the best mode, time and frequency of rehabilitation exercise training for patients after lung cancer surgery. High-quality, multi-center randomized controlled studies are still needed for further practice and exploration. (3) The pulmonary rehabilitation after radical resection of lung cancer needs the coordination and promotion of thoracic surgery medical staff and rehabilitation physiotherapists, psychological counselors, nutritionists and other multidisciplinary, but the current pulmonary rehabilitation mainly focuses on the respiratory function and upper and lower limb function exercise, and the understanding and education of medical staff on pulmonary rehabilitation is insufficient, and the patient's rehabilitation treatment compliance is poor. (4) Preoperative pulmonary rehabilitation can improve the pulmonary function of early patients, optimize the state of the body to increase the opportunity of surgery, if the preoperative and postoperative pulmonary rehabilitation joint implementation may make patients with radical lung cancer surgery benefit more, improve the quality of life, but preoperative pulmonary rehabilitation related clinical studies are few, need to provide theoretical support further research. To sum up, pulmonary rehabilitation applied to thoracoscope lung cancer radical surgery can have a positive effect, by improving the cardiopulmonary function to improve the quality of survival, has great application potential, but still need more high quality, multicenter clinical research and practice with unified specification applies to the lung cancer patients with postoperative pulmonary rehabilitation plan, to speed up the implementation of the pulmonary rehabilitation, application and promotion, effectively regulate recovery postoperatively in patients with lung cancer management, timely access to health.

\section{References}

[1] Bray, F., Ferlay, J., Soerjomataram, I., et al. (2016). Global Cancer Statistics 2018: GLOBOCAN Estimates of Incidence and mortality Worldwide for 36 Canccers in 185 Countries [J]. CA Cancer J Clin., 68(6): 394-424.

[2] Granger, C. (2016). Physiotherapy management of lung cancer [J]. Journal of Physiotherapy, 62(2): 60-67.

[3] Swanson, S. J., Meyers, B. F., Gunnarsson, C. L., et al. (2012). Video assisted reproductive lobectomy is less open and morbid than open lobotomy: a retrospective multi-institutional Database Analysis [J]. AnnThorac Surg, 93(4): 1027-1032.

[4] Zhi Xiuyi, Shi Yuankai, Yu Jinming, et al. (2015). Chinese Guidelines for the Diagnosis and Treatment of primary lung cancer (2015 edition) [J]. Chinese Journal of Cancer, 37(1): 67-78.

[5] Sommer, S., Trier, K., Vibe-petersen, J., et al. (2016). Perioperative Rehabilitation in Operable Lung Cancer Patients (PROLUCA) [J]. Integrative Cancer Therapies, 15(4): 455-466.

[6] Xiao Hui, CUI Yick, Wu Lei, et al. (2008). Research status and progress of pulmonary rehabilitation after lung cancer surgery [J]. Chinese Journal of Gerontology, 38(17): 4312-4314.

[7] Tiep, B., Sun, V., Koczywas, M., et al. (2015). Pulmonary Rehabilitation and Palliative Care for the Lung Cancer Patient [J]. Journal of Hospice \& Palliative Nursing, 17(5): 462-468.

[8] Kim, S. K., Ahn, Y. H., Yoon, J. A., et al. (2015). Efficacy of Systemic drug Rehabilitation After Lung Rehabilitation Medicine [J]. Annals of Rehabilitation Medicine, 39(3): 366.

[9] Saito, H., Hatakeyama, K., Konno, H., et al. (2017). Impact of pulmonary rehabilitation on postoperative complications in patients with lung cancer and chronic obstructive pulmonary disease [J]. Thorac Cancer, 8(5): 451-460.

[10] Spruit, M. A., Singh, S. J., Garvey, C., et al. (2013). An Official American Society/European Respiratory Scoiety Statement: key concepts and advances in pulmonary rehabilitation [J]. Am J Respir Crit Care Med., 188(8): 13-64.

[11] Ren Junlong, Xue Lei, Sun Guangyuan, et al. (2015). Thoracoscopic radical resection of lung cancer in the treatment of early non-small cell lung cancer [J]. Shandong Journal of Medicine, 55(23): 48-50.

[12] Li Lu, Gao Xinyuan, Li Jianhua, et al. (2016). Effect of systemic respiratory training on short-term respiratory and motor function after surgery in patients with lung cancer [J]. Chinese Journal of Rehabilitation Medicine, 31(11): 1225-1229.

[13] Zhang Ye, Huang Bing, Li Yang, et al. (2015). Effects of different distension patterns on pulmonary ventilation and respiratory mechanics after one-lung ventilation. Guangdong Medical Science, 53(19): 2981-2983.

[14] Wu Liu Ping, Cao Su, Gao Yongtao, et al. (2017). Dexmedetomidine can reduce lung injury induced by one lung ventilation during lobectomy [J]. Journal of Clinical Anesthesiology, 33(12): 1171-1173.

[15] Hou Xiaoying, Dong Cuiping, Wang Junhui, et al. (2015). Effect of pulmonary rehabilitation training on preventing respiratory muscle fatigue during mechanical ventilation in patients with lung cancer after operation [J]. Journal of Nursing Education, 33(14): 1299-1301.

[16] Kong Qingqing, Sha Yongsheng, Zhao Yue. (2014). Effect of pulmonary rehabilitation training on quality of life of patients undergoing chemotherapy after lung cancer surgery [J]. Journal of Nursing Refresher, 29(10): 880-882.

[17] Fan Zerong, Xu Zhijie. (2017). Efficacy of systemic pulmonary rehabilitation training after pneumonectomy [J]. China Reha- 
bilitation, 32(01): 23-26.

[18] Liu Lifeng, Sha Yongsheng, Sun Xiaonan, et al. (2017). Effect of rehabilitation training stairway on surgical tolerance of patients with low lung function lung cancer [J]. Tianjin Nursing, 25(02): 113-115.

[19] Chen Xiang, Xu Guxin, Liu Zhen, et al. (2019). Effect of multidisciplinary comprehensive nursing on pulmonary rehabilitation of patients after radical resection of lung cancer [J]. Journal of Guangxi Medical University, 36(07): 1206-1209.

[20] Vagvolgyi, A., Rozgonyi, Z., Kerti, M., et al. (2012). Effectiveness of pulmonary rehabilitation and correlations in between functional parameters, Evaluation of thoracic surgery and severity of post-operative Complications: Randomized Clinical Trial [J]. Journal of Thoracic Disease, 10(6): 3519-3531.

[21] Guo Xintao, Jiao Jianlong, Wei Rongwei, et al. (2008). Effect of rehabilitation training on postoperative lung function of patients undergoing lung cancer surgery [J]. Chinese Journal of Physical Medicine and Rehabilitation, 40(4): 306-308.

[22] Ding Qian. (2019). Effect of pulmonary rehabilitation path on postoperative respiratory function recovery and exercise endurance of patients undergoing radical resection of lung cancer [J]. General Nursing, 17(29): 3674-3676.

[23] Huang Yuxian, Zhang Youwei. (2017). Comparison of the effect of perioperative breathing apparatus and abdominal breathing exercise in patients with lung cancer resection [J]. Journal of Nursing, 32(04): 29-31.

[24] Zhou Min, Zhao Jianping. (2008). Common methods of modern pulmonary rehabilitation [J]. Chinese Journal of Practical Internal Medicine, 38(05): 410-413.

[25] Wang Hao, Liu Bao, Yan An. (2019). Application of diaphragmatic electrical stimulation training in pulmonary rehabilitation of patients after lung cancer surgery [J]. Journal of nursing management, 19(01): 69-72.

[26] He Shengnan, Ni Jingyu, NiuMeie, Tan Minjuan. (2015). Research progress of respiratory function exercise in COPD patients [J]. Journal of nursing advancement, 30(17): 1567-1570.

[27] Han Yan. (2017). Study on the influence of Ba Duan Jin Training on postoperative rehabilitation of patients with non-small cell lung cancer [D]. Shanxi: Shanxi Medical University.

[28] Yang, I. A., Shaw, G., Goddard, J. R., et al. (2016). Use of inhaled corticosteroids in COPD: Improving efficacy [J]. Expert Review of Respiratory Medicine, 17(63): 47-48.

[29] Hu Xianglin, Yang Dong. (2019). Research progress in perioperative optimal management of lung cancer patients with chronic obstructive pulmonary disease (COPD) [J]. Journal of Fudan University (medical edition), 46(02): 267-275.

[30] Qian Lei, Ji Aihua, Zhang Wenjian. (2015). Effect of early enteral nutrition on prevention of postoperative pulmonary infection in patients with lung cancer [J]. Chinese Journal of Nosocomial Infection, 25(02): 415-417.

[31] Jeong, J. H., Yoo, W. G. (2015). Effect of caregiver education on pulmonary rehabilitation, respiratory muscle strength and dyspnea in lung cancer patients [J]. J Phys Ther Sci, 27(6): 1653-1654.

[32] Selzler, A., Wald, J., Sedeno, M., et al. (2017). Telehealth Pulmonary Rehabilitation: A review of the literature and an example of A nationwide initiative to improve the accessibility of pulmonary rehabilitation [J]. Chronic Respiratory Disease, 15(1): 41-47.

[33] Wouters, E. F. M., Wouters, B. B. R. E., Augustin, I. M. L., et al. (2016). Personalised Pulmonary Rehabilitation in COPD [J]. European Respiratory Review, 27(147): 170125.

[34] Rivas-perez, H., Nana-sinkam, P. (2015). Intebral rehabilitation into the undiagnosed management of lung cancer: A review [J]. Respiratory Medicine, 109(4): 447-442.

[35] Lai, Y., Huang, J., Yang, M., et al. (2017). Seven-day intensive preoperative Rehabilitation for elderly patients with lung cancer: a randomized Controlled trial [J]. J Surg Res, 209: 30-36.

[36] Edvardsen, E., Skjonsberg, O. H., Holme, I., et al. (2015). High-intensity Training following Lung Cancer Surgery: A Randomised Controlled Trial [J]. Thorax, 70(3): 244-250.

[37] Chen Ruiying, Liu Ya, Sun Ting, et al. (2019). Effects of pulmonary rehabilitation exercise training on respiratory and motor function, quality of life and total survival of patients with lung cancer [J]. Chinese Journal of Physical Medicine and Rehabilitation, 41(1): 31-36.

[38] Chen Weihai, Xu Bin, Xiong Li, et al. (2015). Meta-analysis on the Effect of pulmonary rehabilitation training on functional recovery of patients with lung cancer [J]. Chinese Journal of Rehabilitation Medicine, 33(01): 76-81.

[39] The sand is immortal, the hole is gentle. The effect evaluation of rapid rehabilitation surgery concept in the nursing application of lung cancer patients [J]. Journal of nurse training, 2017, 32(21): 1963-1965.

[40] Brunelli, A., Kim, A. W., Berger, K. I. (2013). Physiologic evaluation of the patient with lung cancer being considered for resurgery [J]. Chest, 143(5): 166-190.

[41] Qin Mengxia, Qian Yan, Chen Ying. (2019). Advances in the application of pulmonary rehabilitation in the treatment of lung cancer patients [J]. J nursing, 34(10): 101-104.

[42] Kubori, Y., Matsuki, R., Hotta, A., et al. (2017). Com Parison between stair-climbing test and six-minute Walk test after lung Resection using video-assisted tos lobectomy [J]. J Phys Ther Sci, 29(5): 902-904. 
[43] Kubori, Y., Matsuki, R., Hotta, A., et al. (2018). Association between Pulmonary Function and Stair-Climbing Test Results after Lung Study [J]. Canadian Respiratory Journal, 2018: 1-5.

[44] Su Jianhua, Che Guowei. (2017). Status and progress of preoperative pulmonary function assessment in patients with lung cancer [J]. Chinese Journal of Oncology, 44(07): 301-305.

[45] Niu Jinyuan, Li Weiwei, Zhang Wentong. (2015). Current status and research progress of pulmonary function rehabilitation [J]. China Rehabilitation, 30(02): 140-142.

[46] Butov, D., Makieieva, N., Vasylum Chenko, Y., et al. (2015). Interrelationship of Scots Function Parameters in children with Law Asthma in sudden and rate [J]. Advances in Respiratory Medicine, 87: 7-13.

[47] Candemir, I., Ergun, P., Kaymaz, D., et al. (2019). Use of i-bode index to determine efficacy of pulmonary rehabilitation in COPD patients [J]. Tuberk Toraks, 67(2): 116-123. 